Article

\title{
What Enables Individuals to Be More Physically Active across Levels of Frailty?
}

\author{
Dustin Scott Kehler ${ }^{1,2, *}$, Mariana Gonzalez-Lara ${ }^{3}$, Andrea Mayo ${ }^{1}$, \\ Olga Theou ${ }^{1,2}$ \\ 1 School of Physiotherapy, Faculty of Health, Dalhousie University, Halifax, NS \\ B3H 4R2, Canada \\ 2 Division of Geriatric Medicine, Department of Medicine, Dalhousie University, \\ Halifax, NS B3H 2E1, Canada \\ 3 Geriatric Epidemiology Research Department, Instituto Nacional de Geriatria, \\ Mexico 10200, Mexico \\ * Correspondence: Dustin Scott Kehler, Email: scott.kehler@dal.ca; \\ Tel.: +1-902-494-2822.
}

\section{ABSTRACT}

Background: Physical activity is important in the management of frailty. Here we determine if specific frailty deficits relate to lower activity across degrees of frailty, and what types of physical activities are commonly reported.

Methods: Accelerometer data from 6817 adults 20-85 years old (20032004/2005-2006 cycles) of the National Health and Nutrition Examination Survey were analyzed. Moderate-to-vigorous intensity physical activity (MVPA) was measured in 1-minute bouts. Frailty was measured with a 46item frailty index consisting of chronic conditions, healthcare utilization, difficulties in activities of daily living (ADL), and laboratory deficits. Individuals were stratified into frailty groups: non-frail, $<0.10$ (53\%; $n=3610)$; minimally frail, $0.10-0.20(26 \% ; n=1776)$; and frail, $>0.20(21 \%$; $n=1431$ ). Linear regression models adjusted for age, sex, demographics, and accelerometer wear time.

Results: Among the total sample, the presence of individual deficits were generally associated with lower MVPA. The presence of more chronic conditions, ADLs, abnormal laboratory values, and greater healthcare

\section{f Open Access}

Received: 16 December 2019

Accepted: 28 January 2020

Published: 30 January 2020

Copyright (c) 2020 by the author(s). Licensee Hapres, London, United Kingdom. This is an open access article distributed under the terms and conditions of Creative Commons Attribution 4.0 International License. utilization had an independent dose association with lower MVPA. The effect of frailty deficits on MVPA were attenuated when examining individuals by frailty level. The number of ADLs, but not the number of chronic conditions or number of laboratory deficits, were independently associated with a lower MVPA level across frailty levels. Healthcare use was associated with less MVPA in the lower frailty groups. The two most popular reported physical activities were walking and cycling across all frailty levels.

Conclusions: Deficits in ADLs, but not chronic conditions or laboratory deficits, were consistently associated with lower MVPA across frailty levels.

KEYWORDS: frail elderly; accelerometry; physical activity 


\section{ABBREVIATIONS}

ADL, activities of daily living; FI, frailty index; MVPA, Moderate to vigorous intensity physical activity; NHANES, National Health and Nutrition Examination Survey

\section{INTRODUCTION}

Frailty describes the variability in which people age in good or poor health [1]. It arises as a consequence of accumulating health-related deficits over one's lifetime, resulting from damage which goes unrepaired or unremoved across multiple physiologic systems [1]. Frailty is on the rise globally due to an increasingly aging population. Therefore, a response from public health and clinical practice is needed to address its consequential adverse events and burden to the healthcare system [2-4].

Supporting people to live a physically active lifestyle represents one of the most promising strategies to manage frailty $[5,6]$. Despite its health benefits, especially for the increasingly frail [7], people with a higher burden of frailty are less likely engage in moderate to vigorous intensity physical activity (MVPA) recommended for health benefits [8]. The overall number of health deficits are likely a key contributor that hinders an individual's ability to be physically active, however, it remains uncertain if there are specific vulnerabilities which make people more susceptible to be active than others. With this in mind, the objectives of this study are to determine the frailty deficits which are associated with MVPA across levels of frailty, as well as to determine what types of physical activities they report. This information could be valuable for healthcare providers to determine which deficits are most negatively related to inactivity, while also helping to prescribe which physical activities people engage in across levels of frailty to promote healthier aging.

\section{MATERIALS AND METHODS}

Cross-sectional data from the 2003-2004 and 2005-2006 cycles of the National Health and Nutrition Examination Survey (NHANES) were used for this study. Consenting participants recruited into NHANES are a national representation of the United States community-dwelling, noninstitutionalized population. Data were collected by trained personnel for in-home visits to collect demographic and questionnaire data. Medical staff conducted an examination to collect medical, dental, and laboratory measurements. The Institutional Review Board of the Centers for Disease Control and Prevention approved the NHANES study (Protocol \#98-12 (2003-04 cycle); Protocol \#2005-06 (2005-06 cycle)).

\section{Participants}

Individuals 20 years or older who wore an accelerometer were included in this study. Accelerometers were not given to participants if they had ambulatory impairments or other limitations that prevented 
wearing the device. We chose a younger age group because frailty is not always synonymous with older age [9]. Participants were excluded if they had insufficient demographic information, data to calculate a frailty index (FI), and did not wear an accelerometer for at least $10 \mathrm{~h}$ a day for at least 3 days [10].

\section{Physical Activity}

Individuals wore a hip-mounted uniaxial Actigraph (model 7164) accelerometer for seven days to classify minute-by-minute physical activity intensity. Accelerometer counts per minute determined MVPA ( $\geq 2020$ counts per minute) which align with other NHANES studies [11]. MVPA was determined in bouts of at least one minute in accordance with recent physical activity guidelines in the United States [12]. Users were blinded to accelerometer data recording. Trained personnel instructed participants to wear the accelerometer during awake hours and to remove the device if they were bathing or swimming. To determine accelerometer wear time, non-wear time was subtracted from the 24-h day. Non-wear time was defined as $\geq 60$ consecutive minutes of 0 counts/min, but allowed 2 min to reach 1-100 counts/min. The type of physical activities were recorded by self-report.

\section{Frailty}

A 46-item FI developed in NHANES was used and created in accordance with previously published guidelines [13,14]. FI development guidelines recommend that the presence of health-related deficits increase with older age, are not too prevalent $(>80 \%)$ and not too rare $(<1 \%)$. Deficits consisted of chronic conditions, symptoms, activities of daily living (ADL), healthcare use, and laboratory abnormalities. A FI is calculated as a fraction of the total number of health deficits, with scores ranging from 0 to 1. For example, someone with 23/46 deficits would have a FI of 0.5 . Individuals with $<20 \%$ missing individual FI variables were included for analysis. Individuals were stratified into frailty groups: non-frail $(\mathrm{FI}<0.10)$, minimally frail (FI $0.10-0.20)$ and frail $(>0.20)$. These frailty cut-offs are based on our previous research [6,13].

\section{Statistical Analysis}

Descriptive characteristics across levels of frailty are presented as mean (standard error) or frequency (percent) for continuous or categorical variables, respectively. Comparison of MVPA across frailty levels are presented as median (interquartile range) because of nonnormal distribution. Demographic and MVPA across levels of frailty were compared with a one-way analysis of variance for continuous variables and with a Chi-square test for categorical variables. The frequency of participation in the top 10 physical activities were also analyzed across frailty levels. Participation in individual physical activities was defined as 
individuals who self-reported engaging in the physical activity at least once in the past 30 days.

Separate linear regression models were used to determine if individual FI deficits were associated with MVPA for the total sample and then stratified by frailty level. Univariable models were first completed, followed by adjusting for age, sex, education, race, marital status, and accelerometer wear time. The cumulative effects of the number of chronic conditions ( 0 to $\geq 6$ ), ADLs, abnormal laboratory values (all ranged from 0 to 6 or more), and healthcare utilization ( 0 to 3) on MVPA were also completed in unadjusted and fully adjusted linear regression models. Betacoefficients with 95\% confidence intervals are presented for regression modeling. All analyses were performed with SAS 9.4 (SAS Institute, Cary, North Carolina) accounting for survey weights in NHANES.

\section{RESULTS}

\section{Demographics}

From 10,020 participants in the NHANES 2003-04/2005-2006 cycles who were at least 20 years old and wore an accelerometer, 7125 had valid accelerometer data. After removal of individuals without a calculated FI ( $n=298$ ) or missing covariates $(n=10), 6817$ were available for analyses. The excluded sample were slightly younger than the included sample (43.1 vs 47.5 ), were more likely to have a lower education, and less likely to be married, There were no differences in sex, FI, smoking status, or ethnicity between the excluded vs. included sample.

For the included sample, the average age of the sample was 47.5 (0.2), $3504(47.7 \%)$ were female, and the average FI was 0.11(0.01) (Table 1). Participants with higher levels of frailty were older, had a higher proportion of females, were more likely to be former smokers, had a lower education, were less likely to be married, and be Mexican American or Other Hispanic. The proportion of individual FI deficits increased with higher levels of frailty (Supplementary Table S1).

\section{Physical Activity}

Thirty-one percent of participants met the aerobic component of the American Physical Activity Guidelines of 150 min of MVPA per week (Figure 1A). The proportion meeting physical activity guidelines decreased with higher frailty levels, as well as their level of weekly MVPA (Figure 1A,B). Median (interquartile range) for weekly MVPA was 100 (40-199), 141 (71-238), 68 (28-143), and 22 (8-58) for the total sample and the nonfrail, minimally frail, and frail groups, respectively ( $p<0.001$ for group differences). 
Table 1. Demographics by frailty level.

\begin{tabular}{|c|c|c|c|c|c|}
\hline \multirow[t]{2}{*}{ Variable } & \multirow[b]{2}{*}{$\begin{array}{c}\text { Total sample } \\
(n=6817)\end{array}$} & \multicolumn{3}{|c|}{ Frailty level } & \multirow[b]{2}{*}{$P$-value } \\
\hline & & $\begin{array}{c}<\mathbf{0 . 1 0} \\
(n=3610)\end{array}$ & $\begin{array}{l}\mathbf{0 . 1 0}-\mathbf{0 . 2 0} \\
(n=1776) \\
\end{array}$ & $\begin{array}{c}>0.20 \\
(n=1431)\end{array}$ & \\
\hline Age (Mean \pm SE) & $47.5(0.2)$ & $40.5(0.2)$ & $55.1(0.4)$ & $64.1(0.5)$ & $<0.001$ \\
\hline Sex (\% Female) & 3504 (47.7) & $1773(49.7 \%)$ & $962(55.1 \%)$ & 769 (58.2\%) & $<0.001$ \\
\hline Frailty (Mean \pm SE) & $0.11(0.01)$ & $0.04(0.001)$ & $0.14(0.001)$ & $0.29(0.002)$ & $<0.001$ \\
\hline Smoking status (\%) & & & & & $<0.001$ \\
\hline Non-smoker & $3502(50.8 \%)$ & $2024(54.1 \%)$ & $885(48.2 \%)$ & $593(41.7 \%)$ & \\
\hline Former & $1882(26.3 \%)$ & 768 (21.9\%) & $540(30.4 \%)$ & $574(37.6 \%)$ & \\
\hline Current & $1433(22.9 \%)$ & $818(24.0 \%)$ & $351(21.4 \%)$ & $264(20.7 \%)$ & \\
\hline Education (\%) & & & & & $<0.001$ \\
\hline$<$ Grade 12 & $1860(16.5 \%)$ & $782(12.8 \%)$ & $512(18.4 \%)$ & $566(28.1 \%)$ & \\
\hline Highschool/GED & $1667(25.7 \%)$ & $821(23.7 \%)$ & $457(27.2 \%)$ & $389(31.2 \%)$ & \\
\hline Some college & 1939 (32.3\%) & $1132(33.7 \%)$ & 490 (31.7\%) & $317(27.7 \%)$ & \\
\hline Secondary education & $1351(25.5 \%)$ & $875(29.8 \%)$ & 317 (22.6\%) & $159(13.1 \%)$ & \\
\hline Marital status (\%) & & & & & $<0.001$ \\
\hline Married/common law & $4347(67.0 \%)$ & $2426(69.6 \%)$ & $1166(67.4 \%)$ & $755(56.2 \%)$ & \\
\hline Single & $962(14.3 \%)$ & $720(18.5 \%)$ & $164(8.9 \%)$ & $78(5.8 \%)$ & \\
\hline Divorced/separated & $845(12.3 \%)$ & $358(9.8 \%)$ & $244(15.0 \%)$ & $243(17.9 \%)$ & \\
\hline Widowed & $663(6.4 \%)$ & $106(2.1 \%)$ & $202(8.7 \%)$ & $355(20.0 \%)$ & \\
\hline Ethnicity (\%) & & & & & $<0.001$ \\
\hline Mexican American & $1420(7.9 \%)$ & $820(9.3 \%)$ & $360(6.4 \%)$ & $240(4.3 \%)$ & \\
\hline Other Hispanic & $194(3.4 \%)$ & $124(3.7 \%)$ & $47(3.5 \%)$ & $23(2.0 \%)$ & \\
\hline Non-Hispanic Black & $1338(10.2 \%)$ & $689(9.5 \%)$ & 345 (10.6\%) & $304(12.3 \%)$ & \\
\hline Non-Hispanic White & $3589(73.3 \%)$ & 1809 (71.8\%) & $962(74.7 \%)$ & $818(77.0 \%)$ & \\
\hline Other & $276(5.3 \%)$ & $168(5.6 \%)$ & $62(4.9 \%)$ & $46(4.4 \%)$ & \\
\hline
\end{tabular}

Data are presented as survey weighted mean (SE) or survey weighted frequency (\%). $P$-values are the results from a Chi-Square test for comparisons across frailty level.

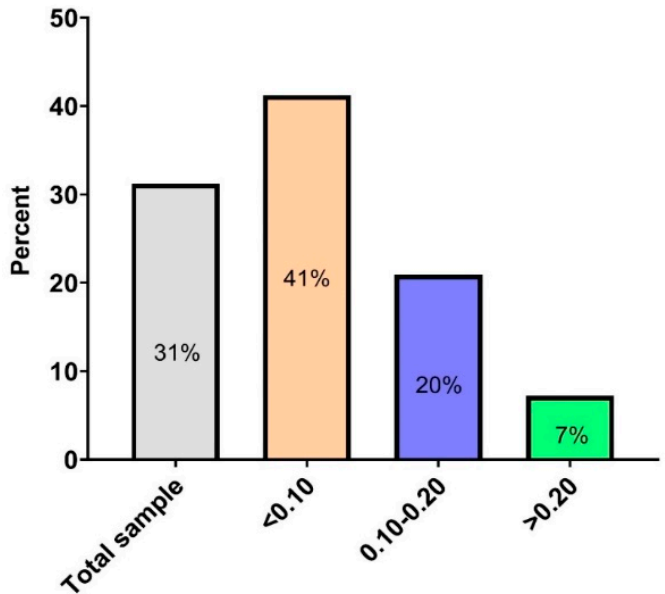

(A)

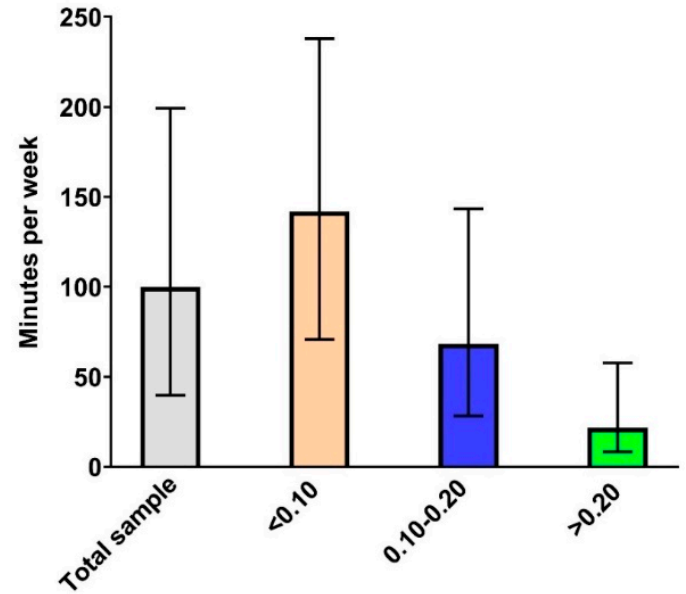

(B)

Figure 1. Physical activity levels. Proportion meeting physical activity guidelines of 150 min per week of MVPA (A) and median (interquartile range) of MVPA per week (B). 


\section{Types of Physical Activities}

The top 10 most reported invidivual physical activities for the total sample and by frailty level can be viewed in Figure 2, Panels A to D. Walking was the most reported physical activity (approximately 30-40\% reported particpating at least once in the last 30 days) for the total sample and all frailty groups. Cycling was the second most reported physical activity in all groups. Interestingly, there was a small percentage in all frailty groups who reported weight lifting at least once (Total sample: 9.8\% (ranked 4th); Non-frail: 11.9\% (ranked 3rd); minimally frail 5.5\% (ranked 9th); Frail: 3.9\% (ranked 6th)).

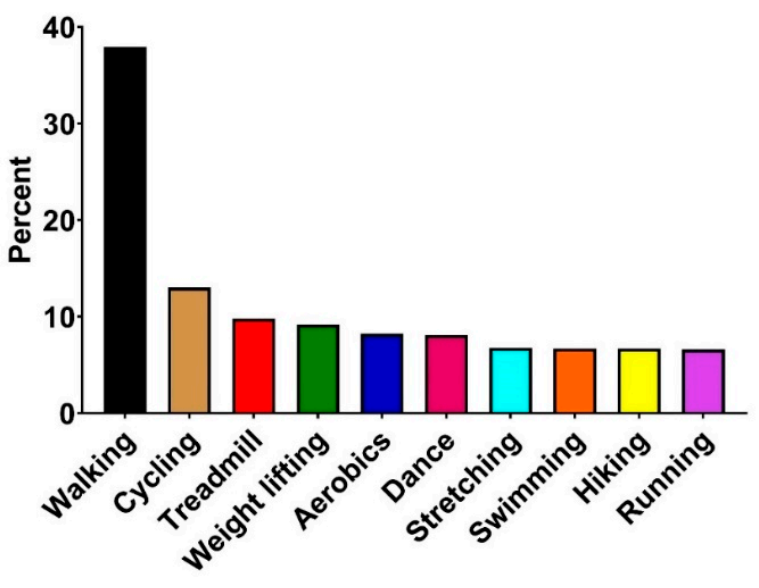

(A)

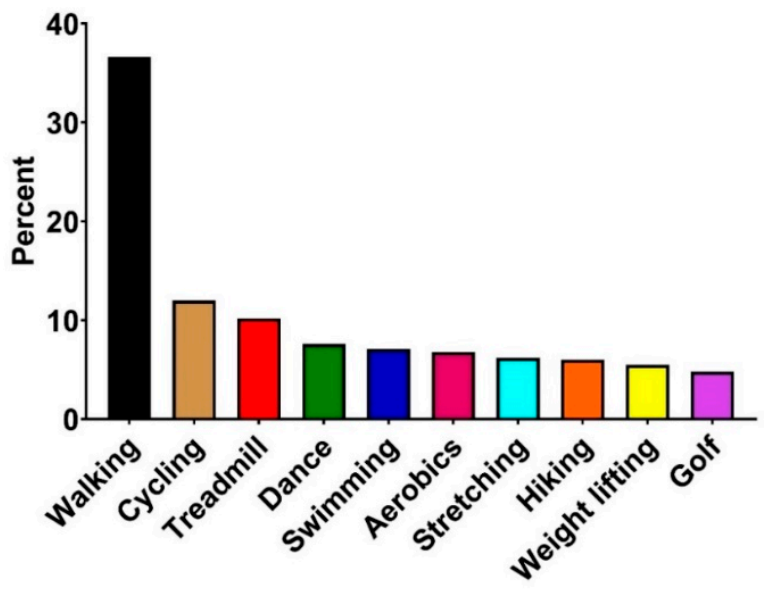

(C)

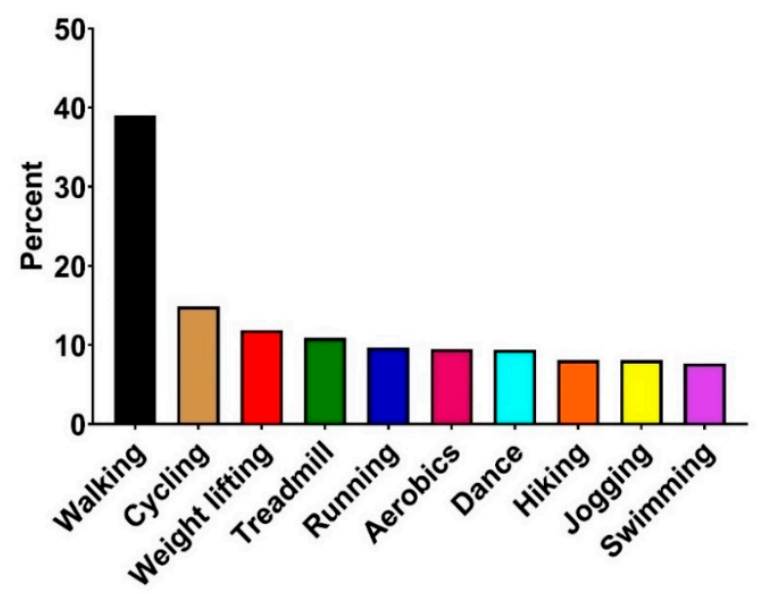

(B)

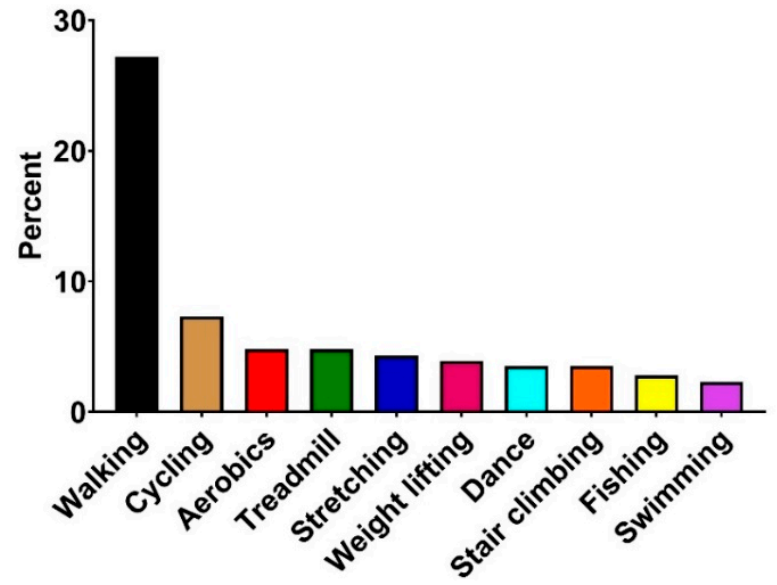

(D)

Figure 2. The top 10 most reported physical activities across levels of frailty. Data are survey-weighted prevalence for the total sample (A), and non-frail (B), minimally frail (C), and frail (D).

\section{Association of Demographics and Individual Frailty Deficits with Physical Activity}

Simple linear regression and multivariable linear regression models adjusting for age, sex, race, education, marital status, and accelerometer wear time were completed, modeling weekly MVPA with demographics (Table 2) and individual FI deficits for the total sample and by levels of 
frailty (Figure 3A-I; Supplementary Table S2). Here we present fully adjusted models only.

\section{Demographics}

Increasing age was significantly associated with lower MVPA levels in the total sample (Table 2). Compared to females, males were more physically active. Former or non-smokers were more physically active than current smokers. Secondary education was associated with a higher MVPA level compared to those who did not graduate. Marital status was not associated with MVPA. Non-Hispanic Black and White, as well as "other" ethnic groups were less active than Mexican Americans. Across frailty levels, older age was consistently associated with less MVPA, whereas males consistently had higher MVPA compared to females. Nonsmokers and former smokers were more active than current smokers for the non-frail and minimally frail groups, but not the frail group. There was no effect of education or marital status on MVPA across frailty groups. Non-Hispanic Black and White groups, as well as the "other" group compared to Mexican Americans, were less active in the non-frail group only. Race had no effect in the minimally frail group, whereas the Other Hispanic group were more active when compared to Mexican Americans.

Table 2. Association of demographic variables with MVPA by frailty level.

\begin{tabular}{|c|c|c|c|c|}
\hline \multirow[t]{2}{*}{ Variable } & \multirow[b]{2}{*}{$\begin{array}{c}\text { Total sample } \\
\quad(n=6817)\end{array}$} & \multicolumn{3}{|c|}{ Frailty level } \\
\hline & & $\begin{array}{c}<\mathbf{0 . 1 0} \\
(n=3610)\end{array}$ & $\begin{array}{l}\mathbf{0 . 1 0}-\mathbf{0 . 2 0} \\
(n=1776)\end{array}$ & $\begin{array}{c}>0.20 \\
(n=1431)\end{array}$ \\
\hline Age (per 10 years) & $\begin{array}{c}-29.4 \\
(-31.7,-27.01)\end{array}$ & $\begin{array}{c}-19.9 \\
(-23.9,-15.8)\end{array}$ & $\begin{array}{c}-20.6 \\
(-24.7,-16.4)\end{array}$ & $\begin{array}{c}-17.5 \\
(-23.6,-11.4)\end{array}$ \\
\hline Sex (ref: Female) & $\begin{array}{c}69.9 \\
(62.5,77.3) \\
\end{array}$ & $\begin{array}{c}86.3 \\
(76.2,96.4) \\
\end{array}$ & $\begin{array}{c}45.3 \\
(32.5,58.2) \\
\end{array}$ & $\begin{array}{c}22.3 \\
(11.1,33.5)\end{array}$ \\
\hline \multicolumn{5}{|l|}{ Smoking status (\%) } \\
\hline Non-smoker & $\begin{array}{c}17.9 \\
(8.7,27.1)\end{array}$ & $\begin{array}{c}17.0 \\
(4.7,29.4)\end{array}$ & $\begin{array}{c}13.9 \\
(2.7,30.5)\end{array}$ & $\begin{array}{c}10.8 \\
(-3.8,25.4)\end{array}$ \\
\hline Former & $\begin{array}{c}16.4 \\
(6.2,26.8)\end{array}$ & $\begin{array}{c}19.6 \\
(4.9,34.4)\end{array}$ & $\begin{array}{c}19.6 \\
(1.1,38.0)\end{array}$ & $\begin{array}{c}8.4 \\
(-6.7,23.5)\end{array}$ \\
\hline Current & Reference & Reference & Reference & Reference \\
\hline \multicolumn{5}{|l|}{ Education (\%) } \\
\hline$<$ Grade 12 & Reference & Reference & Reference & Reference \\
\hline Highschool/GED & $\begin{array}{c}-5.2 \\
(-15.8,5.3)\end{array}$ & $\begin{array}{c}-16.9 \\
(-34.5,0.6)\end{array}$ & $\begin{array}{c}-0.1 \\
(-18.6,18.5)\end{array}$ & $\begin{array}{c}0.4 \\
(-10.8,11.6)\end{array}$ \\
\hline Some college & $\begin{array}{c}-7.3 \\
(-17.9,3.4)\end{array}$ & $\begin{array}{c}-16.6 \\
(-34.1,0.9)\end{array}$ & $\begin{array}{c}-15.9 \\
(-33.1,1.3)\end{array}$ & $\begin{array}{c}10.9 \\
(-1.7,23.5)\end{array}$ \\
\hline Secondary education & $\begin{array}{c}23.2 \\
(11.1,35.2) \\
\end{array}$ & $\begin{array}{c}11.6 \\
(-7.5,30.6) \\
\end{array}$ & $\begin{array}{c}14.9 \\
(-4.6,34.4) \\
\end{array}$ & $\begin{array}{c}2.9 \\
(-11.2,17.1)\end{array}$ \\
\hline
\end{tabular}


Table 2. Cont.

\begin{tabular}{|c|c|c|c|c|}
\hline \multirow[t]{2}{*}{ Variable } & \multirow[b]{2}{*}{$\begin{array}{c}\text { Total sample } \\
\quad(n=6817)\end{array}$} & \multicolumn{3}{|c|}{ Frailty level } \\
\hline & & $\begin{array}{c}<\mathbf{0 . 1 0} \\
(n=3610)\end{array}$ & $\begin{array}{l}\mathbf{0 . 1 0 - 0 . 2 0} \\
(n=1776)\end{array}$ & $\begin{array}{c}>0.20 \\
(n=1431)\end{array}$ \\
\hline \multicolumn{5}{|l|}{ Marital status (\%) } \\
\hline Married/common law & $\begin{array}{c}-0.1 \\
(-10.6,10.5)\end{array}$ & $\begin{array}{c}-5.9 \\
(-21.7,9.8)\end{array}$ & $\begin{array}{c}-2.1 \\
(-22.5,18.4)\end{array}$ & $\begin{array}{c}2.4 \\
(-8.6,13.4)\end{array}$ \\
\hline Single & $\begin{array}{c}2.1 \\
(-11.9,16.1)\end{array}$ & $\begin{array}{c}2.7 \\
(-16.4,21.8)\end{array}$ & $\begin{array}{c}0.9 \\
(-26.8,28.6)\end{array}$ & $\begin{array}{c}17.6 \\
(-9.2,44.5)\end{array}$ \\
\hline Divorced/separated & Reference & Reference & Reference & Reference \\
\hline Widowed & $\begin{array}{c}6.7 \\
(-6.6,20.1)\end{array}$ & $\begin{array}{c}-8.6 \\
(-39.1,21.9) \\
\end{array}$ & $\begin{array}{c}-7.1 \\
(-30.5,16.3)\end{array}$ & $\begin{array}{c}9.1 \\
(-7.5,25.6)\end{array}$ \\
\hline \multicolumn{5}{|l|}{ Ethnicity (\%) } \\
\hline Mexican American & Reference & Reference & Reference & Reference \\
\hline Other Hispanic & $\begin{array}{c}16.2 \\
(-9.1,41.5)\end{array}$ & $\begin{array}{c}13.6 \\
(-19.0,46.2)\end{array}$ & $\begin{array}{c}28.3 \\
(-17.6,74.1)\end{array}$ & $\begin{array}{c}40.8 \\
(0.1,81.4)\end{array}$ \\
\hline Non-Hispanic Black & $\begin{array}{c}-29.3 \\
(-42.3,-16.3)\end{array}$ & $\begin{array}{c}-30.1 \\
(-48.0,-12.1)\end{array}$ & $\begin{array}{c}-8.2 \\
(-29.0,12.6)\end{array}$ & $\begin{array}{c}-7.8 \\
(-25.0,9.4)\end{array}$ \\
\hline Non-Hispanic White & $\begin{array}{c}-20.7 \\
(-32.5,-8.8)\end{array}$ & $\begin{array}{c}-19.4 \\
(-35.2,-3.5)\end{array}$ & $\begin{array}{c}-11.9 \\
(-30.4,6.7)\end{array}$ & $\begin{array}{c}-9.0 \\
(-25.9,7.9)\end{array}$ \\
\hline Other & $\begin{array}{c}-46.9 \\
(-64.9,-28.9)\end{array}$ & $\begin{array}{c}-53.1 \\
(-78.0,-28.3)\end{array}$ & $\begin{array}{c}-25.9 \\
(-54.1,2.3)\end{array}$ & $\begin{array}{c}-13.6 \\
(-36.5,9.3)\end{array}$ \\
\hline
\end{tabular}

Data are B-coefficient (95\% CI) for a fully adjusted model (age, sex, race, marital status, ethnicity, and accelerometer wear time). Data highlighted in bold are statistically significant $(p<0.05)$.

\section{Chronic conditions}

In general, the presence of any chronic condition was independently associated with less MVPA in the total sample (Figure 3A; Supplementary Table S2) except for the presence of a thyroid condition, cancer, or kidney problems. There was a dose-response association between lower MVPA and a greater number of chronic conditions, with $\beta$-coefficient estimates ranging from -11.7 (95\% CI: $-21.1,-2.2)$ for one condition, to $-47.8(-69.2$, -26.4) for six or more conditions compared to no conditions (Figure 3B). When separate models were completed by frailty level, the effect of individual and number of deficits on MVPA were largely mitigated. A previous heart attack in the non-frail group, diabetes in the minimally frail group, and arthritis in the frail group, were independently related to lower weekly MVPA. The number of chronic conditions were not associated with MVPA any frailty level group. 


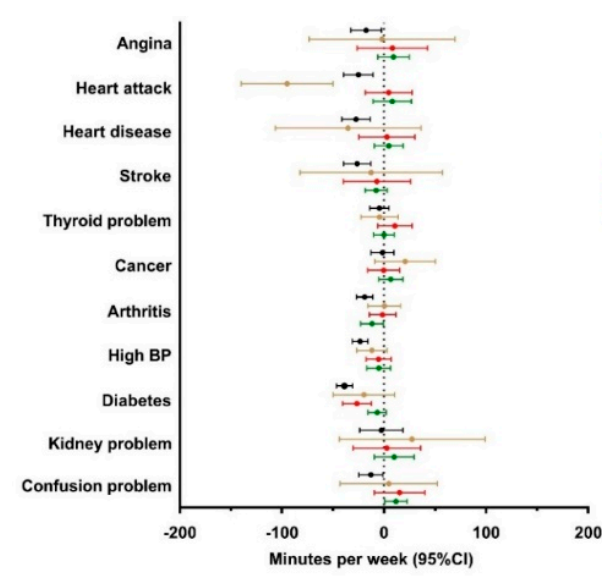

(A)

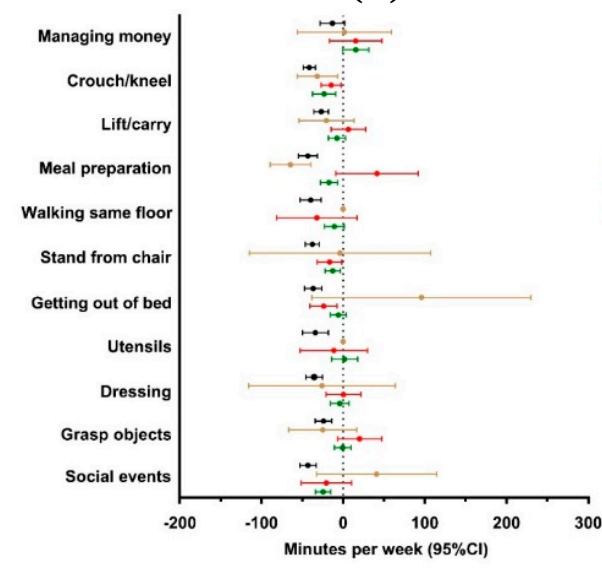

(C)

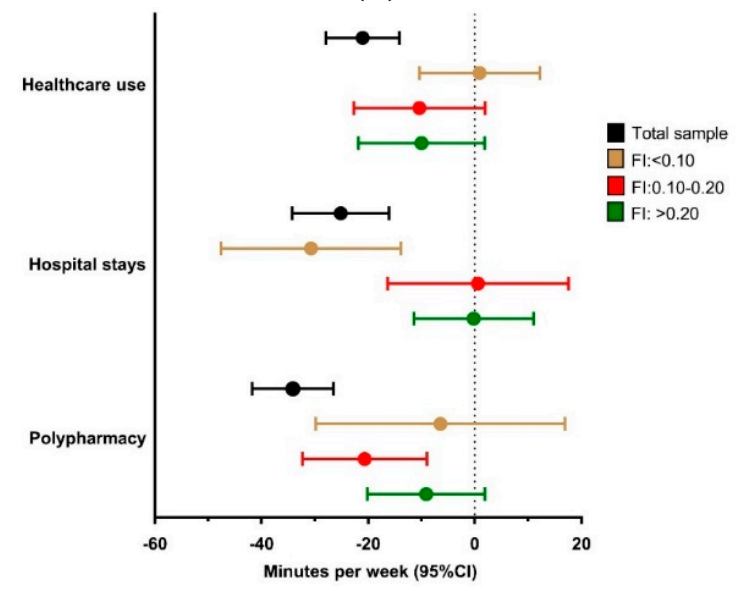

(E)

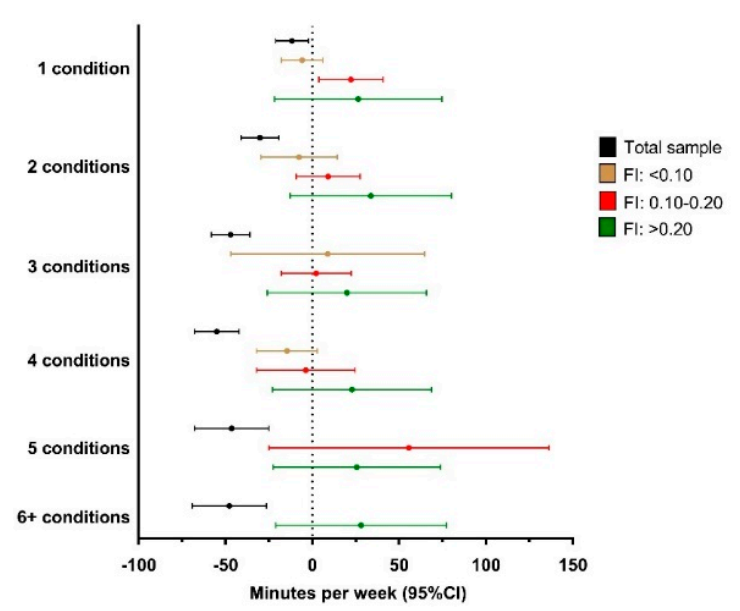

(B)

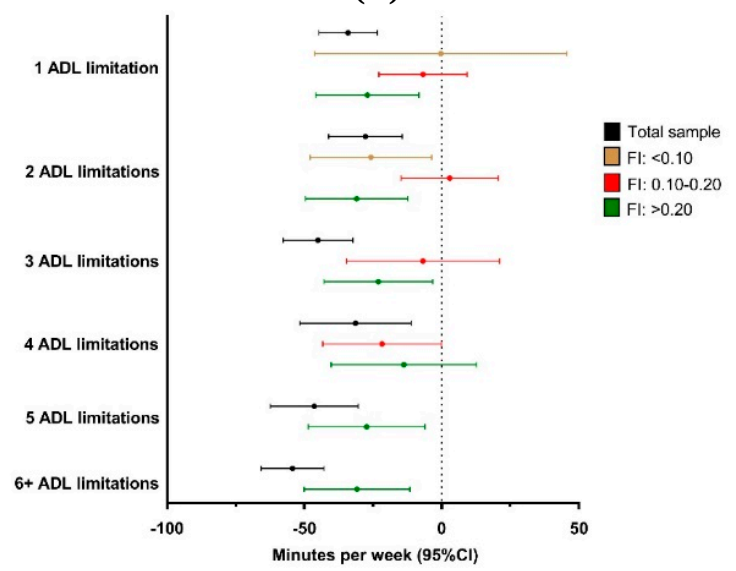

(D)

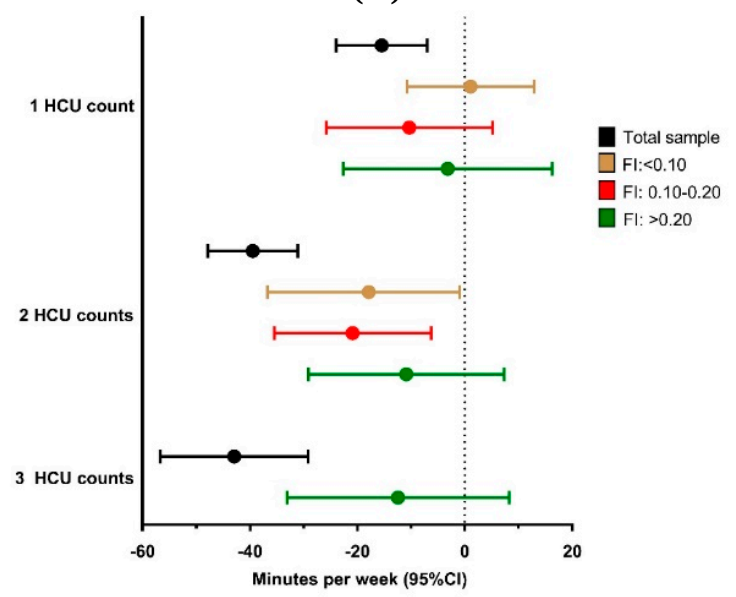

(F)

Figure 3. Associations of individual frailty index deficits with participation in moderate to vigorous intensity physical activity. Data are by chronic conditions (A), chronic condition count (B); ADL difficulties (C) and their count (D); healthcare utliziation (E) and their count (F); and lab abnormalities (G) and the total number of abnormal values (H). 


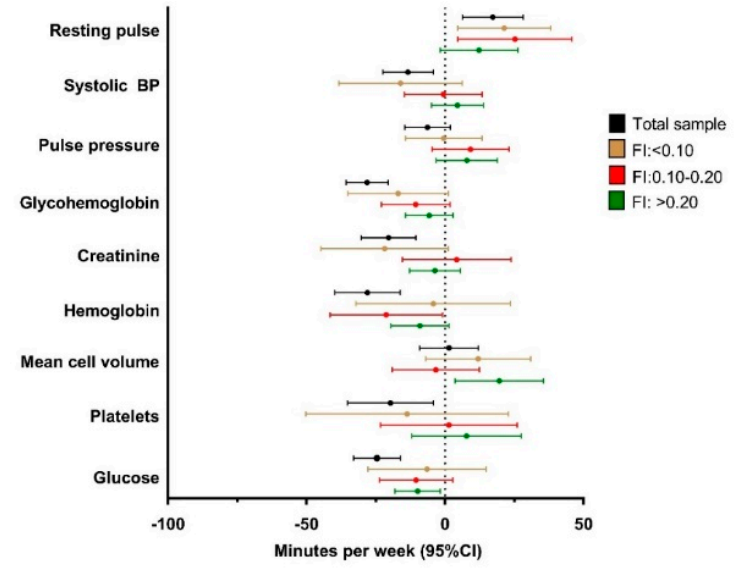

(G)

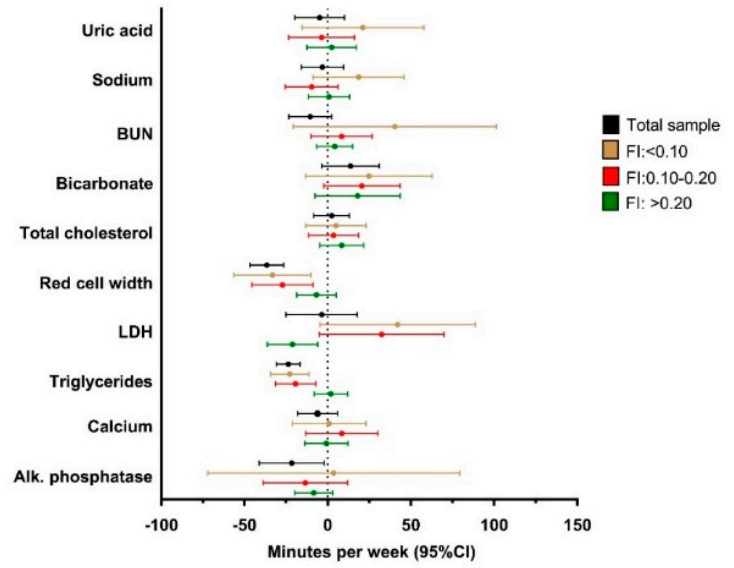

(H)

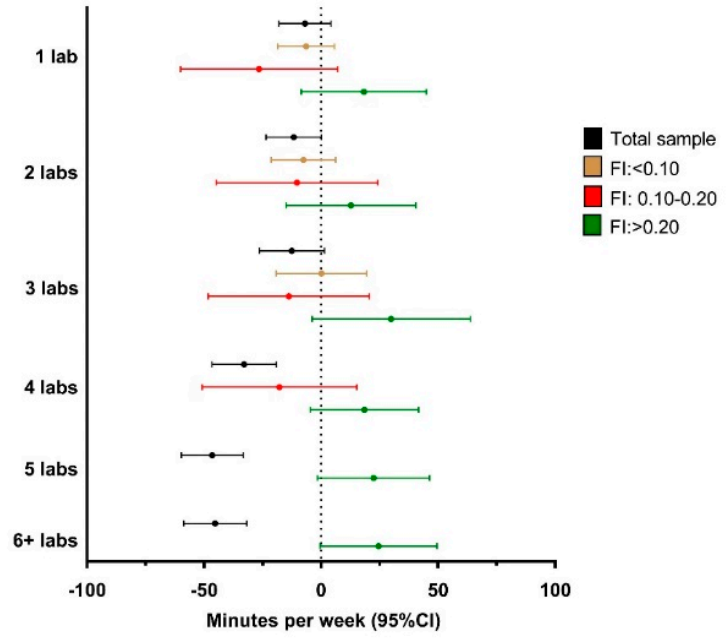

(I)

Figure 3. Cont.

\section{ADL difficulties}

Among the total sample, the presence of any ADL difficulty, except with managing money, was detrimentally associated with MVPA (Figure 3C; Supplementary Table S2). Difficulties ranging from having one (-34.0 $(-44.7,-23.4))$ to the presence of six or more $(-54.3(-65.7,-42.8))$ was less pronounced as comparfed to chronic conditions in relation to a doserelationship with lower MVPA compared to having no difficulties in the total sample and in all frailty groups (Figure 3D; Supplementary Table S2). Similar to the effect of chronic conditions on MVPA, the relationship between difficulties on MVPA were in large part mitigated. Difficulties with crouching/kneeling or stooping were consistently associated with lower MVPA across levels of frailty. For the non-frail group, difficulties with preparing meals and using a fork or knife had a strong association with lower levels of MVPA. Difficulties standing from an armless chair (minimally frail and frail), and getting in and out of bed (minimally frail only), and difficulties with preparing meals (non-frail and frail) were associated with less weekly MVPA. 


\section{Healthcare utilization}

All healthcare utilization deficits (frequency of healthcare use, overnight hospital stays, polypharmacy; Figure 3E; Supplmentary Table S2), as well as the total number of healthcare use deficits, were associated with lower levels of MVPA amongst the entire sample (Figure 3F; Supplementary Table S2). Frequency of healthcare use was not associated with MVPA in any frailty group. Overnight hospital stays in the non-frail group and polypharmacy in the minimally frail group were independently associated with lower habitual MVPA; whereas no healthcare utilization variables related to physical activity in the frail group. The total count of healthcare use deficits were associated with lower weekly MVPA in the non-frail and minimally frail groups only.

\section{Laboratory abnormalities}

For the total sample, abnormal values with systolic blood pressure, glycohemoglobin, platelet count, glucose, red cell distribution width, triglycerides, alkaline phosphatase, creatinine, and hemoglobin were associated with less weekly MVPA (Figure 3G,H; Supplementary Table S2). Abnormal values in resting heart rate was associated with higher MVPA. A higher number of abnormal laboratory values, starting at four or more, was associated with less MVPA (Figure 3I; Supplementary Table S2). Abnormal resting heart rate in the non-frail and vulnerable frailty group, as well as mean cell volume in the mildly frail group, was associated with higher MVPA levels. Abnormal glycohemoglobin (non-frail), fasting glucose (frail), red cell distribution width (non-frail, minimally frail), lactate dehydrogenase (frail), triglycerides (non-frail, minimally frail), and hemoglobin (minimally frail) were detrimentally associated with less weekly MVPA. Laboratory counts were not associated with MVPA when stratified by frailty.

\section{DISCUSSION}

The purpose of our study was to determine if individual frailty deficits were related to MVPA when graded by frailty severity, and to determine what types of physical activities were most commonly reported in these groups. When analyzing the entire study sample, we showed that the presence of most deficits, with the exception of approximately half of laboratory deficits, were associated with an inactive lifestyle. However, when we accounted for different levels of frailty, ADL difficulties showed the strongest relationship with lower MVPA, whereas there was no relationship with the number of chronic conditions or laboratory deficits and MVPA. Walking and cycling were the highest reported physical activities across the frailty groups. Collectively, this information may inform public health and clinical practice to support individuals to be more physically active across all frailty levels. 
The most frequently reported physical activities were aerobic in nature across all frailty level groups (Figure 2). Specifically, walking and cycling were the two most popular physical activities, which corroborates with previous findings [15]. Although only a small fraction of individuals reported weightlifting, it was among the top 10 physical activities reported despite frailty status. Public health and clinical recommendations should encourage more resistance-based exercises to address frailty. Emerging evidence from clinical trials suggest that combining resistance exercises with aerobic physical activity may have the greatest benefit to managing frailty levels compared to either in isolation [6]. However, resistance exercises have generally been prescribed at low intensity, which may not be as effective as higher intensity resistance training. A further understanding of the impact of resistance exercises on frailty is needed to establish its efficacy.

Individual deficits were shown to have a more widespread effect on MVPA levels when the total sample was analyzed. In particular, a greater accumulation of chronic conditions, ADL difficulties, healthcare utilization, and laboratory abnormalities, had a dose relationship with lower MVPA levels (Figure 3B,D,F,I; Supplementary Table S2). In contrast, the relationship between FI deficits and MVPA were generally mitigated when analyses were completed across frailty level. These data somewhat conflict with previous findings. For example, individuals from the UK Biobank who wore accelerometers were shown to have less weekly MVPA if they had almost any of the 147 chronic diseases examined [16]. We also showed that participants in NHANES 50 years or older accumulated approximately 50 fewer minutes (106 $\mathrm{min}$ vs $66 \mathrm{~min}$ ) for those with cardiovascular disease compared to those without [17]. However, these previous studies did not stratify their analyses by frailty level. The total accumulation of deficits, regardless of a specific health problem, may account for the attenuated relationship with individual deficits and MVPA when we graded frailty. Indeed, a possible explanation may be that MVPA levels are simply lower as frailty severity increases (Figure 1A,B) which may make the strength of individual deficits on an inactive lifestyle less prominent. This idea supports the notion that what matters the most is knowing how many things are wrong with someone rather than individual health problems [18].

However, there was a consistent relationship between individual FI deficits and MVPA across frailty grades with ADL limitations. Difficulties with crouching, kneeling, or stooping, and a greater number of ADL deficits, revealed a persistent relationship with lower physical activity levels. Although the present study could not identify a temporal relationship between physical activity and ADL limitations due to its crosssectional nature, it is possible that maintaining or increasing physical activity has a greater impact on physical function. Specifically, analysis of the Lifestyle Interventions and Independence or Elders study, which was a 24-month aerobic exercise intervention study compared to health 
education, demonstrated a lower rate of major mobility disability for participants who increased their physical activity level [19].

The focus of our study was to examine the relationship with FI deficits with MVPA, however these findings need to be tested further. It is possible that there are age and sex differences with respect to differences in the strength of relationships between different aspects of deficits and MVPA. Specifically, there is a stronger relationship with MVPA on frailty in males compared to females [20] and we showed that males accumulated more MVPA than females (Table 1). The effect of individual frailty deficits on sedentary behaviors, which are characterized by low energy expenditure in a seated or lying posture [21], should also be explored because they are demonstrated to be independently associated with higher frailty levels from that of physical activity [22,23]. There is also a stronger relationship between frailty with physical inactivity and high sedentary levels, therefore individual frailty deficits may influence physical inactivity and sedentary behavior in meaningful ways beyond investigation of movement behaviors (sedentary time and physical activity) in isolation [24,25]. The effect of health deficits on other health behaviors such as nutrition is also a worthwhile area of inquiry [26,27].

Our study has several strengths and limitations. A strength of this study was the use of a nationally representative sample of community-dwelling adults, making the findings here generalizable to a large proportion of the United States population. Furthermore, the use of accelerometry in conjunction with self-reported individual physical activities provides a more precise estimate of physical activity levels while also understanding the types of physical activities which were the most frequently reported. However, there were several physical activities reported which are not accurately captured by the uniaxial accelerometer used in NHANES such as cycling, weightlifting and swimming [28]. Therefore, the true amount of MVPA may be underestimated here. The temporal relationship between physical activity and frailty also cannot be determined, due to the crosssectional nature of NHANES. Therefore, it is unclear whether higher frailty levels resulted in lower physical activity levels or vice-versa. Lastly, we used standardized accelerometer cut-points used in NHANES to classify MVPA to increase the generalizability of our findings; however, this approach may misclassify physical activity levels in older and frailer adults. However, it is difficult to classify a specific cut-point given the range of abilities of individuals.

\section{CONCLUSIONS}

Our study provides insights into the relationship between individual frailty deficits on MVPA. Addressing ADL limitations rather than chronic conditions or laboratory abnormalities may support community-dwelling individuals to engage in a more physically active lifestyle when frailty is more severe. These findings should be further tested in a longitudinal 
study which capture objective physical activity behaviors in conjunction with details on individual physical activities.

\section{SUPPLEMENTARY MATERIAL}

The supplementary material is available online at https://doi.org/10.20900/agmr20200007.

\section{AUTHOR CONTRIBUTIONS}

DSK and OT designed the study. DSK performed the data analysis. All authors contributed to writing/revising the paper and consent to submit the manuscript.

\section{CONFLICTS OF INTEREST}

The authors declare that they have no conflicts of interest.

\section{REFERENCES}

1. Mitnitski AB, Mogilner AJ, Rockwood K. Accumulation of deficits as a proxy measure of aging. ScientificWorldJournal. 2001;1:323-36.

2. Hoogendijk EO, Afilalo J, Ensrud KE, Kowal P, Onder G, Fried LP. Frailty: implications for clinical practice and public health. Lancet. 2019;394(10206):1365-75.

3. Kojima G, Iliffe S, Walters K. Frailty index as a predictor of mortality: a systematic review and meta-analysis. Age Ageing. 2018;47(2):193-200.

4. Han L, Clegg A, Doran T, Fraser L. The impact of frailty on healthcare resource use: a longitudinal analysis using the Clinical Practice Research Datalink in England. Age Ageing. 2019;48(5):665-71.

5. Puts MTE, Toubasi S, Andrew MK, Ashe MC, Ploeg J, Atkinson E, et al. Interventions to prevent or reduce the level of frailty in community-dwelling older adults: a scoping review of the literature and international policies. Age Ageing. 2017;46(3):383-92.

6. Kehler DS, Theou O. The impact of physical activity and sedentary behaviors on frailty levels. Mech Ageing Dev. 2019;180:29-41.

7. Godin J, Blodgett JM, Rockwood K, Theou O. Replacing Sedentary Time with Light or Moderate-Vigorous Physical Activity Across Levels of Frailty. J Aging Phys Act. 2019:1-19.

8. Blodgett J, Theou O, Kirkland S, Andreou P, Rockwood K. The association between sedentary behaviour, moderate-vigorous physical activity and frailty in NHANES cohorts. Maturitas. 2015;80(2):187-91.

9. Kehler DS, Ferguson T, Stammers AN, Bohm C, Arora RC, Duhamel TA, et al. Prevalence of frailty in Canadians 18-79 years old in the Canadian Health Measures Survey. BMC Geriatr. 2017;17(1):28.

10. Heesch KC, Hill RL, Aguilar-Farias N, van Uffelen JGZ, Pavey T. Validity of objective methods for measuring sedentary behaviour in older adults: a systematic review. Int J Nutr Phys Act. 2018;15(1):119. 
11. Troiano RP, Berrigan D, Dodd KW, Masse LC, Tilert T, McDowell M. Physical activity in the United States measured by accelerometer. Med Sci Sport Exerc. 2008;40(1):181-8.

12. Committee PAGA. Physical Activity Guidelines Advisory Committee Report, 2008. Washington, DC. (US): U.S. Department of Health and Human Services; 2008.

13. Theou O, Blodgett JM, Godin J, Rockwood K. Association between sedentary time and mortality across levels of frailty. CMAJ. 2017;189(33):E1056-64.

14. Searle SD, Mitnitski A, Gahbauer EA, Gill TM, Rockwood K. A standard procedure for creating a frailty index. BMC Geriatr. 2008;8:24.

15. Dai S, Carroll DD, Watson KB, Paul P, Carlson SA, Fulton JE. Participation in Types of Physical Activities Among US Adults--National Health and Nutrition Examination Survey 1999-2006. J Phys Act Health. 2015;12(Suppl 1):S128-40.

16. Barker J, Smith Byrne K, Doherty A, Foster C, Rahimi K, Ramakrishnan R, et al. Physical activity of UK adults with chronic disease: cross-sectional analysis of accelerometer-measured physical activity in 96706 UK Biobank participants. Int J Epidemiol. 2019;48(4):1167-74.

17. Kehler DS, Clara I, Hiebert B, Stammers AN, Hay JL, Schultz A, et al. The association between patterns of physical activity and sedentary time with frailty in relation to cardiovascular disease. Aging Med. 2019;2(1):18-26.

18. Cesari M, Marzetti E, Thiem U, Perez-Zepeda MU, Abellan Van Kan G, Landi F, et al. The geriatric management of frailty as paradigm of "The end of the disease era”. Eur J Intern Med. 2016;31:11-4.

19. Mankowski RT, Anton SD, Axtell R, Chen SH, Fielding RA, Glynn NW, et al. Device-Measured Physical Activity As a Predictor of Disability in MobilityLimited Older Adults. J Am Geriatr Soc. 2017;65(10):2251-6.

20. Kehler DS, Clara I, Hiebert B, Stammers AN, Hay JL, Schultz A, et al. Sexdifferences in relation to the association between patterns of physical activity and sedentary behavior with frailty. Arch Gerontol Geriatr. 2019;87:103972.

21. Tremblay MS, Aubert S, Barnes JD, Saunders TJ, Carson V, Latimer-Cheung AE, et al. Sedentary Behavior Research Network (SBRN)—Terminology Consensus Project process and outcome. Int J Behav Nutr Phys Act. 2017;14(1):75.

22. Kehler DS, Hay JL, Stammers AN, Hamm NC, Kimber DE, Schultz ASH, et al. A systematic review of the association between sedentary behaviors with frailty. Exp Gerontol. 2018;114:1-12.

23. Kehler DS, Clara I, Hiebert B, Stammers AN, Hay JL, Schultz A, et al. The association between bouts of moderate to vigorous physical activity and patterns of sedentary behavior with frailty. Exp Gerontol. 2018;104:28-34.

24. da Silva VD, Tribess S, Meneguci J, Sasaki JE, Garcia-Meneguci CA, Carneiro JAO, et al. Association between frailty and the combination of physical activity level and sedentary behavior in older adults. BMC Public Health. 2019;19(1):709.

25. Del Pozo-Cruz B, Manas A, Martin-Garcia M, Marin-Puyalto J, Garcia-Garcia FJ, Rodriguez-Manas L, et al. Frailty is associated with objectively assessed sedentary behaviour patterns in older adults: Evidence from the Toledo Study for Healthy Aging (TSHA). PLoS One. 2017;12(9):e0183911. 
26. Whittaker AC, Asamane EA, Aunger JA, Bondarev D, Cabbia A, Gensous N, et al. Physical Activity and Nutrition INfluences in Ageing: Current Findings from the PANINI Project. Adv Geriatr Med Res. 2019;1:e190005. https://doi.org/10.20900/agmr20190005

27. Jayanama K, Theou O, Blodgett JM, Cahill L, Rockwood K. Frailty, nutritionrelated parameters, and mortality across the adult age spectrum. BMC Med. 2018;16(1):188.

28. Howe CA, Staudenmayer JW, Freedson PS. Accelerometer prediction of energy expenditure: vector magnitude versus vertical axis. Med Sci Sport Exerc. 2009;41(12):2199-206.

How to cite this article:

Kehler DS, Gonzalez-Lara M, Mayo A, Theou O. What Enables Individuals to Be More Physically Active across Levels of Frailty? Adv Geriatr Med Res. 2020;2(1):e200007. https://doi.org/10.20900/agmr20200007 